\title{
INCLUSÃO NA EDUCAÇÃO INFANTIL NA CIDADE DE CAXIAS DO SUL/RS: CONTATO INICIAL COM O LÓCUS DE PESQUISA
}

\section{Inclusion in early childhood education in Caxias do Sul / RS: initial contact with research place}

\author{
Milena Elizabeth Vieira - Centro Universitário da Serra Gaúcha - FSG, Brasil \\ Indianara Sehaparini - Centro Universitário da Serra Gaúcha - FSG, Brasil \\ Éderson Jacinto de Oliveira- Centro Universitário da Serra Gaúcha - FSG, Brasil \\ Mariana Galimberti Lopes - Centro Universitário da Serra Gaúcha - FSG, Brasil \\ Tatiele Jacques Bossi - Centro Universitário da Serra Gaúcha - FSG, Brasil
}

RESUMO: A educação infantil para crianças com deficiência é um direito legal. Devem ser respeitadas as particularidades de cada criança, sendo proposta uma educação de qualidade. O presente estudo tem como objetivo apresentar o contato inicial com o lócus de pesquisa sobre a inclusão de bebês e crianças com deficiência na educação infantil na cidade de Caxias do Sul/RS. A aquisição dos dados referentes à inclusão nesta cidade foi realizada através do contato com a Secretaria Municipal de Educação de Caxias do Sul (SMED). As informações obtidas no contato com a SMED foram analisadas através de análise quantitativa descritiva. O município em estudo gerencia 45 escolas de educação infantil, dessas, 14 atendem um total de 25 crianças com deficiência. Desse total de bebês e crianças inclusas, $68 \%(\mathrm{n}=17)$, estão na préescola; $28 \%(\mathrm{n}=7)$ frequentam o maternal, e apenas $4 \%(\mathrm{n}=1)$ frequentam o berçário. Os diagnósticos que apresentam predominância são de autismo, paralisia cerebral, transtornos de ordem neurológica e síndrome de Down, em ordem decrescente. $\mathrm{O}$ aumento de bebês e crianças com deficiência nas escolas reforça o olhar e a atenção de entidades públicas para esses espaços. Por isto, é importante a pesquisa neste contexto, a fim de garantir o papel protetivo destes ambientes inclusivos para esse público.

Palavras-chave: Educação Inclusiva. Educação Infantil. Lócus de Pesquisa.

ABSTRACT: Early childhood education for children with disabilities is a legal right. The particularities of each child must be respected, being proposed a quality education. This study aims to present the initial contact with the research locus about the inclusion of babies and children with disabilities in early childhood education in the city of Caxias do Sul/RS. The acquisition of data regarding inclusion in this city was made through contact with the Municipal Education Secretariat of Caxias do Sul (SMED). The information obtained from the contact with SMED was analyzed through descriptive quantitative analysis. The city under study manages 45 preschools, of which 14 serve a total of 25 children with disabilities. Of this total of babies and children included, 68\% $(\mathrm{n}=17)$ are in kindergarten; 28\% $(\mathrm{n}=7)$ attend the nursery school at age 34 , and only $4 \%(\mathrm{n}=1)$ attend the nursery at age $0-2$. The predominant diagnoses are autism, cerebral palsy, neurological disorders and Down syndrome, in decreasing order. The rise of infants and children with disabilities in schools reinforces the public eye and

Educação, Psicologia e Interfaces, Volume 3, Dossiê Inclusão e Diversidade, p. 111-121, 2019.

ISSN: 2594-5343. DOI: https://doi.org/10.37444/issn-2594-5343.v3i4.196 
attention of these spaces. Therefore, research in this context is important in order to ensure the protective role of these inclusive environments for this public.

Keywords: Inclusive Education. Early Childhood Education. Locus of Research.

\section{INTRODUÇÃO}

A deficiência é uma condição que se faz presente em $2,79 \%$ da população infantil, considerando as idades de 0 a 4 anos, o que representa aproximadamente 5 milhões de crianças (SDH/PR, 2012). Observando a magnitude desse dado em níveis nacionais pode-se pensar a deficiência por diferentes olhares: como uma questão de saúde pública, que necessita de atenção multiprofissional; como um aspecto da assistência social, em termos de defesa de direitos; e também como uma particularidade a ser discutida em todos os âmbitos da educação. Esse processo engloba diferentes políticas públicas, diferentes profissionais e é caracterizado por um atendimento em rede. Entretando, o exercício efetivo da inclusão pode apresentar dificuldades, uma vez que todos os âmbitos citados são importantes e precisam estar em constante harmonia para abarcar as necessidades desse público, com deficiência (PEREIRA; TEIXEIRA, 2013).

A educação infantil é defendida na Constituição Federal de 1988 como um direito de todos. A Constituição vem reforçar o que na década de 40 foi apresentado na Declaração dos Direitos Humanos, no segundo inciso, do artigo XXI, que coloca o direito de acesso igual às políticas públicas para todos (ONU, 1948). Também no sentido legal, um documento significativo na defesa de direitos para pessoas com deficiência é a Declaração de Salamanca (UNESCO, 1994), que discorre sobre o direito à educação, de forma inclusiva. Esta defende as particularidades de todas as crianças e a importância destas serem respeitadas em sua individualidade, sem perder o olhar da qualidade de educação. Dessa forma, a educação infantil, também deve ser ofertada de modo a estimular a aprendizagem, com a compreensão de que cada criança tem o tempo e a forma de aprender dela. Além disso, esse documento traz o conceito de que todas as crianças devem ter a diversidade protegida com o intuito de diminuir os comportamentos sociais discriminatórios. Frente a isso, as escolas passaram a atender demandas diferentes das que eram de costume e com esse processo surgem 
Inclusão na educação infantil na cidade de Caxias do Sul/RS: contato inicial com o lócus de pesquisa

problemáticas e dificuldades em relação às mudanças práticas dessas determinações teóricas (UNESCO, 1994).

A busca pela melhoria da qualidade referente aos recursos e estratégias pedagógicos, como também as relações estabelecidas no universo escolar, devem estar presentes na educação escolar inclusiva (SEKKEL; ZANELATTO; BRANDÃO, 2010). Os estudos relacionados à inclusão nos ambientes educacionais tendem a compreender como esse conceito se dá na prática. Alguns estudos apontam a forma com a qual os professores têm manejado o processo inclusivo. Outras discussões dicorrem sobre como os educadores são preparados para lidar com os alunos e como podem repensar práticas pedagógicas para que aconteça a qualificação do ensino para todas as crianças. Ainda, as pesquisas mostram formas de manejo familiar, para que as famílias possam compreender os direitos delas e como estas podem participar aticamente na inclusão dos filhos, auxiliando-os nesse processo. (PASSARINHO, 2015; ALMEIDA et al, 2011. MARQUES et al., 2017; TELES et al., 2013). Todavia, o que pode ser percebido é que a maioria dos estudos sobre os ambientes educacionais inclusivos se referem ao ensino regular, sendo baixo o número de estudos voltados à educação infantil.

É no momento da educação infantil que a criança vivência as primeiras experiências recreativas com seus pares, estando fora do ambiente familiar. Assim, as investigações de interação social no período pré-escolar se tornam importantes (CAMARGO; BOSA, 2009). A educação infantil é uma construção recente no contexto brasileiro. A família e os grupos sociais eram os responsáveis pela educação das crianças, antes destas adentrarem as instituições de ensino. Contudo, com a globalização e a permanência das mulheres no mercado de trabalho, criou-se uma necessidade de espaços onde as crianças pudessem se desenvolver e crescer de modo saudável, desde os primeiros meses de vida. Nesse contexto, surgem as creches e as pré-escolas, que atualmente, em muitos momentos, são os responsáveis pelos cuidados dos bebês e crianças pequenas (ALVES, 2018; CHAVES; SILVA; CAVALCANTE, 2018).

O que tem provocado discussões na literatura é o fato de como estes espaços têm incluído crianças que necessitam de cuidados especiais. Por ser na infância o espaço onde os sujeitos se constituem psiquicamente e começam a desenvolver as habilidades sociais deles, os contextos de inserção infantil precisam compreender as singularidades,

Educação, Psicologia e Interfaces, Volume 3, Dossiê Inclusão e Diversidade, p. 111-121, 2019.

ISSN: 2594-5343. DOI: https://doi.org/10.37444/issn-2594-5343.v3i4.196 
bem como educar estes sujeitos a respeitar as diferenças (ALVES, 2018; CHAVES; SILVA; CAVALCANTE, 2018). Entretanto, bebês e crianças com deficiência possuem estigmas, que em diversos momentos caracterizam esses sujeitos como incapazes, criando assim, uma rejeição social prévia (ALVES, 2018).

Por mais que hoje exista a garantia do acesso à educação infantil para as crianças com deficiência, são diversos os problemas que ainda se apresentam para a inclusão efetiva e assertiva deste público. Esses impasses vão desde a estrutura física do ambiente escolar, até a falta de capacitação dos profissionais que atendem as crianças. Portanto, é importante a construção de espaços e práticas pedagógicas que auxiliem no desenvolvimento da singularidade do sujeito. Aos educadores, cabe a tarefa de atender as reais necessidades dos bebês e das crianças, contudo isso nem sempre é possível, devido ao número de alunos que estes precisam cuidar. Isso pode acarretar na educação infantil uma padronização de ensino, sendo que esse espaço pode servir apenas como uma preparação para o ensino regular (ALVES, 2018).

Para compreender melhor a inclusão na etapa de educação infantil é importante que sejam realizadas pesquisas que acessem os bebês e as crianças com deficiência, bem como os educadores que estão em contato com estas crianças. A criação de políticas pedagógicas efetivas apenas é possível com a escuta das demandas dos profissionais que se deparam com os impasses da inclusão deste público na escola. Frente a isso, o objetivo do presente estudo é apresentar o contato inicial com o lócus de pesquisa sobre a inclusão de bebês e crianças com deficiência na educação infantil, na cidade de Caxias do Sul/RS.

\section{MATERIAL E MÉTODO}

O Núcleo de Pesquisa em Psicologia Educacional e do Desenvolvimento NuPPED iniciou no ano de 2018 como um grupo de pesquisa do Curso de Psicologia da FSG Centro Universitário. O projeto do núcleo intitulado "Experiências de educadoras frente à inclusão de crianças com deficiência na educação infantil: contribuições dos conceitos winnicottianos" foi aprovado pelo Comitê de Ética em Pesquisa da FSG (CAAE 99716718.1.0000.5668). O projeto tem como objetivo investigar as experiências de educadoras frente à inclusão de crianças com deficiência na educação infantil, a partir de conceitos da teoria de Winnicott (1965/1993), tais como holding, 
Inclusão na educação infantil na cidade de Caxias do Sul/RS: contato inicial com o lócus de pesquisa

manuseio, apresentação de objetos, previsibilidade, adaptação ao saber materno e diagnóstico pedagógico.

Nesse projeto, a forma elegida para coleta de dados foi através de entrevistas com educadoras e para tanto, entrou-se em contato com a Secretaria de Educação de Caxias do Sul (SMED), a fim de obter informações sobre as escolas de educação infantil que tinham crianças com deficiência em processo inclusivo. Através desse contato inicial, a SMED enviou uma lista de escolas que atendem bebês e crianças com deficiência, em que consta a faixa etária dos mesmos, bem como seu diagnóstico. Com isso, o presente estudo faz uma análise documental dessas informações fornecidas pela SMED a fim de apresentar as particularidades do público-alvo da pesquisa. A análise documental é utilizada quando se busca compreender os dados de um material específico, de preferência, dados internos de instituições (GIL, 2018).

\section{RESULTADOS}

Os dados obtidos para o presente estudo foram submetidos à análise quantitativa descritiva, a fim de apresentar o contato inicial com o lócus de pesquisa sobre a inclusão de bebês e crianças com deficiência na educação infantil, na cidade de Caxias do Sul/RS. No contato com a SMED, os dados recebidos apontaram que o município gerencia 45 escolas de educação infantil. Desses ambientes, apenas 32\% $(n=14)$, têm bebês e crianças com deficiência incluídas no quadro de alunos, sendo que se beneficiam dos serviços da educação infantil do município, um total de 25 indivíduos. Os dados fornecidos também apontam que as crianças maiores têm mais adesão do que as crianças menores, considerando que o número maior de crianças se encontra na préescola, que atende faixas etárias dos 4 aos 6 anos de idade (17 crianças, representando $68 \%$ do total). O maternal, que corresponde a faixa etária de 3 a 4 anos, atende 7 crianças com deficiência, o que gera o percentual de $28 \%$. E por fim, como dado mais preocupante, aparece o berçário (0 a 2 anos) com a inclusão de apenas uma criança (4\%), conforme representado na figura 1 .

Educação, Psicologia e Interfaces, Volume 3, Dossiê Inclusão e Diversidade, p. 111-121, 2019.

ISSN: 2594-5343. DOI: https://doi.org/10.37444/issn-2594-5343.v3i4.196 


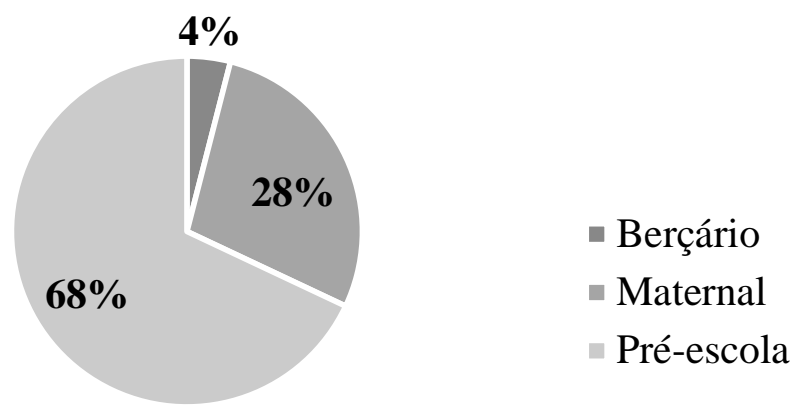

Figura 1 - Número de crianças matriculadas na educação infantil em escolas gerenciadas pela SMED de Caxias do $\mathrm{Sul} / \mathrm{RS}$

Outro aspecto que pode ser identificado é referente aos diagnósticos das crianças matriculadas nas escolas. Ocorre um predomínio de diagnóstico de autismo (28\%), seguido dos diagnósticos de paralisia cerebral (16\%); transtornos de ordem neurológica, como irritabilidade cerebral neonatal e hemorragia intracraniana (12,5\%); e síndrome de Down (6\%), em percentuais mais elevados. Ainda, foi possível observar diagnósticos que ocorrem em situações únicas, sendo estes, deformidade congênita do pé; retardo no desenvolvimento neuropsicomotor; síndrome de Turner; transtorno do desenvolvimento de fala e linguagem; transtorno específico do desenvolvimento motor; e perda auditiva. Frente aos dados, também se encontrou que $19 \%$ dos diagnósticos se encontram indefinidos, aguardando pela avaliação de uma equipe multiprofissional. Também, pode ser extraída a informação de que do total de 32 diagnósticos informados pela SMED, seis casos (24\%) apresentaram comorbidades, por exemplo, autismo e síndrome de Down.

\section{DISCUSSÃO}

Frente a estes dados, pode-se pensar que há muito a se avançar quanto ao cumprimento das leis que garantem o acesso à inclusão de bebês e crianças com deficiência nas creches e escolas de educação infantil. Ainda mais quando se sabe que muitos municípios brasileiros não conseguem proporcionar ambientes educacionais de qualidade para recepcionar esse público. Isso acarreta na falta de suporte para o desenvolvimento infantil destes indivíduos, sendo este, mais um agravante para os 
Inclusão na educação infantil na cidade de Caxias do Sul/RS: contato inicial com o lócus de pesquisa

bebês e crianças com deficiência que possam se encontram em situação vulnerável (VITTA, et al. 2018).

A cidade de Caxias do Sul, no último censo do IBGE (2010), era composta por 435.564 habitantes. Por ser a segunda maior cidade do Estado do Rio Grande do Sul, é possível presumir que o número de habitantes no município hoje esteja maior. Assim, os dados fornecidos pela SMED sobre os números de bebês e crianças com deficiência matriculados nas escolas de educação infantil são alarmantes, já que expõem a baixa adesão desse público nesses espaços. Isso permite os questionamentos: Onde estão os bebês com deficiência do município? Por que o número de crianças com deficiência matriculadas nas escolas infantis é baixo?

Por meio dos questionamentos, pode ser pensada a hipótese de que esses bebês e crianças estejam sob os cuidados familiares integralmente. É possível também presumir que a família possa sentir uma sobrecarga perante aos cuidados desses indivíduos. Isso pode acarretar, muitas vezes, no atraso do desenvolvimento das potencialidades dos bebês e das crianças com deficiência, por não receberem os estímulos proporcionados pelos demais ambientes, para além do familiar. Também, é possível supor que não há a inclusão deste público devido ao desconhecimento das famílias sobre o acesso e o funcionamento dos ambientes educacionais infantis. Ainda, a falta de recursos humanos para o atendimento desse público e a falta de materiais que auxiliem nas atividades das crianças com deficiência são dificuldades impostas na inserção destas na escola (VITTA, et al. 2018).

É através do desenvolvimento das potencialidades que o sujeito consegue superar as dificuldades. $\mathrm{O}$ estigma social relacionado à criança com deficiência tende a colocá-la em uma posição de "não saber". Assim, a escola é a instituição que pode auxiliar na identificação de potencialidades dos bebês e das crianças com deficiência. Os ambientes educacionais infantis desenvolvem a tarefa de ajudar a família a compreender as características destes sujeitos. As relações entre a família e a escola permitem o desenvolvimento da aprendizagem desses bebês e crianças, bem como favorecem o processo inclusivo. Assim, destaca-se a importância da educação infantil para os bebês e crianças com deficiência, para que a família consiga identificar as

Educação, Psicologia e Interfaces, Volume 3, Dossiê Inclusão e Diversidade, p. 111-121, 2019.

ISSN: 2594-5343. DOI: https://doi.org/10.37444/issn-2594-5343.v3i4.196 
competências e as habilidades desses sujeitos, a partir das evoluções educacionais (BOSSI, 2017).

Ainda, é relevante destacar a variedade de diagnósticos das crianças atendidas nas escolas de educação infantil de Caxias do Sul, sendo um aspecto corroborado pelos estudos até então produzidos. Vários estudos, como ressaltado na revisão de literatura de Vitta et al. (2018), acabam por se utilizar de bebês e crianças com diferentes tipos de deficiência em suas análises, justamente por ser um público difícil de ser acessado na educação infantil. Isso pode sobrecarregar os educadores no processo de inclusão, visto que cada criança exige particularidades específicas de manejo no contexto escolar infantil, devido à deficiência dela. No entanto, ressalta-se que para inclusão efetiva dos bebês e das crianças na etapa de educação infantil, o educador precisa ver para além da deficiência da criança, de modo a ter uma visão das particularidades de manejo necessárias para aquele sujeito. O mais importante é que esse profissional se relacione e se vincule com o bebê ou a criança, e não com a deficiência destes, promovendo a inclusão a partir da experiência com essa criança, e não necessariamente através de um saber prévio da educadora sobre os diferentes tipos de deficiência (BOSSI, 2017).

Para encerrar, vale destacar que devido aos números expressivamente baixos de bebês e crianças incluídas na educação infantil no município de Caxias do Sul/RS, bem como os aspectos discutidos sobre a importância desses públicos acessarem os serviços educacionais, destaca-se a importância da realização de mais pesquisas nessa área. Propõe-se que investigar sobre as experiências de educadoras frente à inclusão é relevante para qualificar o processo inclusivo.

Por fim, cabe ressaltar algumas limitações deste estudo. Os dados acessados foram através do gerenciamento público do município de Caxias do Sul, de modo que os autores não tiveram acesso ao número de crianças incluídas em escolas privadas de ensino. Com isso, cabe a investigação nesse campo a fim de se ter um perfil completo sobre a inclusão na educação infantil em Caxias do Sul/RS.

\section{CONSIDERAÇÕES FINAIS}

Os estigmas sociais e os aspectos socioculturais do ambiente em que a criança com deficiência está inserida influenciam diretamente no processo de inclusão educacional. A entrada e permanência dos bebês e das crianças nas escolas faz com que esse público receba maior atenção de entidades pública. Isso propicia o 
Inclusão na educação infantil na cidade de Caxias do Sul/RS: contato inicial com o lócus de pesquisa

desenvolvimento de melhorias no atendimento educacional. Além disso, no momento em que a família descobre que tem um bebê com deficiência, esta passa a ter que lidar com carga emocional do estigma social da deficiência. O círculo familiar presencia assim um luto pelo filho ideal e, consequentemente, esse momento delicado pode ser um complicador no acesso à educação infantil deste sujeito.

Considerando o papel protetivo do ambiente educacional, coloca-se a importância de fomentar reflexões e pesquisas nesse campo, a fim de que sejam qualificadas as políticas educacionais de inclusão. Por meio destes avanços, as famílias dos bebês com deficiência poderão se sentir mais seguras para ocupar os espaços de direito nos ambientes educacionais. Assim, os bebês e as crianças com deficiência podem ter acesso a mais um ambiente protetivo que fortaleça os processos de desenvolvimento deles. Para maior efetividade na inclusão infantil, também é importante que profissionais qualificados estejam presentes nos ambientes educacionais.

A psicologia é um campo que têm muito a ofertar nos processos inclusivos. A presença de psicólogos educacionais nos ambientes de ensino pode proporcionar aos educadores maior segurança no manejo de bebês e crianças com deficiências. Devido a isso, é importante que sejam ampliadas as pesquisas nos contextos de educação infantil. Com o presente estudo, foi possível compreender que, em Caxias do Sul/RS, os espaços de educação infantil ainda são poucos frequentados pelo público que necessita de inclusão. Isso pode ser um dos motivos pelos quais estudos que abrangem esse público são difíceis de serem produzidos.

\section{REFERÊNCIAS BIBLIOGRÁFICAS}

ALVES, Fabíola Fernanda Patrocínio. A inclusão das crianças com deficiência na educação infantil: processo em construção. Educação, Porto Alegre, v. 41, n. 2, p. 270279, Maio/ago., 2018.

BRAZ-AQUINO, Fabíola de Sousa; FERREIRA, Ingrid Rayssa Lucena;

CAVALCANTE, Lorena de Almeida. Concepções e Práticas de Psicólogos Escolares e Docentes acerca da Inclusão Escolar. Psicologia: Ciência e Profissão, Brasília, v. 36, n. 2, p. 255-266, jun. 2016.

BOSSI, T. J. Inclusão de bebê com deficiência física em creche: programa de acompanhamento para educadoras com base em conceitos Winnicottianos. Porto Alegre: UFRGS, 2017. Tese (Doutorado em Psicologia) Programa de Pós-graduação em Psicologia, Universidade Federal do Rio Grande do Sul, 2017.

Educação, Psicologia e Interfaces, Volume 3, Dossiê Inclusão e Diversidade, p. 111-121, 2019.

ISSN: 2594-5343. DOI: https://doi.org/10.37444/issn-2594-5343.v3i4.196 
CAMARGO, Síglia Pimentel Höher; BOSA, Cleonice Alves. Competência social, inclusão escolar e autismo: revisão crítica da literatura. Psicologia \& sociedade, v. 21, n. 1, p. 65-74, 2009.

CHAVES, Jéssica Regina; SILVA, Priscila Ferreira da; CAVALCANTE, Ana Célia Sousa. "Na essência somos iguais, na diferença nos respeitamos": Estágio em Psicologia Escolar. Psicologia Escolar e Educacional., Maringá, v. 22, n. 3, p. 643645, dez. 2018.

GIL, Antonio Carlos. Como elaborar projetos de pesquisa. 6. ed. São Paulo: Atlas, 2018 .

INSTITUTO BRASILEIRO DE GEOGRAFIA E ESTATÍSTICA. Censo Demográfico Populacional 2010: resultados gerais da amostra. Rio de Janeiro: IBGE, 2010.

MARQUES, Heloisa et al. Percepção de professores e gestores de educação sobre a inclusão de crianças com deficiência visual. SALUSVITA, Bauru, v. 36, n. 1, p. 7-21, 2017.

PASSARINHO, Isabel. Em rede fazemos a diferença»: um percurso de aprendizagem, empowerment e inclusão educativa e comunitária com pais de crianças e jovens com NEE. Lusíada. Intervenção Social, Lisboa, n. 42/45, p. 191-205. 2015.

PEREIRA, Karine Yanne de Lima.; TEIXEIRA, Solange Maria. Redes e intersetorialidade nas políticas sociais: reflexões sobre sua concepção na política de assistência social. Textos \& Contextos, Porto Alegre, v. 12, n. 1, p. 114 - 127, jan./jun. 2013.

SECRETARIA DE DIREITOS HUMANOS DA PRESIDÊNCIA DA REPÚBLICA. Cartilha do Censo 2010: pessoas com deficiência. Brasília: SDH/PR, 2012.

SEKKEL, Marie Claire; ZANELATTO, Raquel; BRANDÃO, Suely de Barros. Uma questão para a educação inclusiva: expor-se ou resguardar-se?. Psicologia Ciência e Profissão, v. 30, n. 2, p. 296-307, 2010.

TELES, Fernanda Moreira; RESEGUE, Rosa; PUCCINI, Rosana Fiorini. Habilidades funcionais de crianças com deficiências em inclusão escolar: barreiras para uma inclusão efetiva. Ciência \& Saúde Coletiva, Rio de Janeiro, v. 18, n. 10, p. 3023-3031, out. 2013.

ORGANIZAÇÃO DAS NAÇÕES UNIDAS. Declaração dos Direitos Humanos. Paris: ONU, 1948.

ORGANIZAÇÃO DAS NAÇÕES UNIDAS PARA A EDUCAÇÃO, A CIÊNCIA E A CULTURA. Declaração de Salamanca e linha de ação sobre necessidades educativas especiais. Genebra: UNESCO, 1994.

VITTA, Fabiana Cristina Frigieri de et al. A Produção Científica Nacional na Área de Educação Especial e a Creche. Revista Brasileira de Educação Especial, Bauru, v. 24, n. 4, p. 619-636, dez. 2018.

Credenciais da/os autora/es 
Inclusão na educação infantil na cidade de Caxias do Sul/RS: contato inicial com o lócus de pesquisa

VIEIRA, Milena Elizabeth. Graduanda em Psicologia, Centro Universitário da Serra Gaúcha (FSG). E-mail: milena.vieira200@gmail.com

SEHAPARINI, Indianara. Graduanda em Psicologia, Centro Universitário da Serra Gaúcha (FSG). E-mail: indianara.sehaparini@gmail.com

OLIVEIRA, Éderson Jacinto de. Graduando em Psicologia, Centro Universitário da Serra Gaúcha (FSG). E-mail: eder.tocchetto@ gmail.com

LOPES, Mariana Galimberti. Graduanda em Psicologia, Centro Universitário da Serra Gaúcha (FSG). E-mail: mgalimbertilopes@gmail.com

BOSSI, Tatiele Jacques. Mestra, Doutora e Pós-Doutora em Psicologia pelo Programa de PósGraduação em Psicologia da Universidade Federal do Rio Grande do Sul (UFRGS). Professora do Curso de Psicologia do Centro Universitário da Serra Gaúcha (FSG). E-mail: tatiele.bossi@fsg.edu.br

Endereço para correspondência: Milena Elizabeth Vieira. Rua Os Dezoito do Forte, n. 2366, CEP: 95020-472, Caxias do Sul/RS. E-mail: milena.vieira200@ gmail.com

Como citar este artigo (Formato ABNT): VIEIRA, Milena Elizabeth et al.. Inclusão na educação infantil na cidade de Caxias do Sul/RS: contato inicial com o lócus de pesquisa. Educação, Psicologia e Interfaces, v. 3, Dossiê Inclusão e Diversidade, p. 111-121, 2019. DOI: https://doi.org/10.37444/issn-2594-5343.v3i4.196

Recebido: $27 / 08 / 2019$.

Aceito: 20/11/2019.

Educação, Psicologia e Interfaces, Volume 3, Dossiê Inclusão e Diversidade, p. 111-121, 2019.

ISSN: 2594-5343. DOI: https://doi.org/10.37444/issn-2594-5343.v3i4.196 